\title{
A Primary Peritoneal Serous Carcinoma Metastasizing to the Stomach Mimicking a Submucosal Tumor: A Potential Pitfall of Clinical Diagnosis
}

\author{
Mayumi Kobayashia, c, Hiroshi Yoshida ${ }^{\mathrm{b}}$, Shun-ichi Ikeda ${ }^{\mathrm{a}}$, Reiko Watanabe ${ }^{\mathrm{b}}$, \\ Tomoyasu Kato
}

\begin{abstract}
A primary peritoneal serous carcinoma (PPSC) usually presents as peritoneal dissemination. We report a case of gastric metastasis from PPSC mimicking a primary gastric submucosal tumor (SMT). A 62-year-old woman presented to our hospital with abundant ascites, omental caking, and normal-sized ovaries. Cellblock analysis of ascite specimens indicated high-grade serous Müllerian carcinoma. Gastrointestinal endoscopy detected a 4-cm SMT, which was not gastric cancer, on the gastric antrum. A clinical diagnosis of PPSC was rendered. After chemotherapy, the gastric SMT reduced in size. Following this, the patient underwent complete resection of the residual tumors including gastric SMT. Pathological examination revealed that the gastric lesion was metastasis from PPSC. The assumption that an SMT lesion is a benign primary gastric tumor not requiring surgical resection may hinder optimal tumor debulking. Therefore, preoperative biopsy should be considered, if the situation allows.
\end{abstract}

Keywords: Gastric metastasis; Primary peritoneal serous carcinoma; Submucosal tumor

\section{Introduction}

A primary peritoneal serous carcinoma (PPSC) is a pelvic serous carcinoma (which includes ovarian and tubal serous carcinoma) and usually presents as peritoneal dissemination. There are no reports of gastric metastasis from PPSC in the English literature. Here, we report a case of gastric metastasis from PPSC mimicking a primary gastric submucosal tumor

Manuscript accepted for publication October 10, 2016

aDivision of Gynecology, National Cancer Center Hospital, Tokyo, Japan bDepartment of Pathology and Clinical Laboratories, Pathology Division, National Cancer Center Hospital, Tokyo, Japan

${ }^{\mathrm{c} C o r r e s p o n d i n g ~ A u t h o r: ~ M a y u m i ~ K o b a y a s h i, ~ D i v i s i o n ~ o f ~ G y n e c o l o g y, ~ N a t i o n-~}$ al Cancer Center Hospital, 5-1-1 Tsukiji, Chuo-ku, Tokyo 104-0045, Japan. Email: maykobay@ncc.go.jp

doi: http://dx.doi.org/10.14740/jmc2664w
(SMT). Gynecologists should recognize and identify this unusual pattern of metastasis to achieve complete tumor resection. Furthermore, preoperative gastric biopsy should be considered to judge the indication for surgical resection.

\section{Case Report}

A 62-year-old woman presented at her neighborhood hospital with complaints of abdominal distension. Transvaginal ultrasonography showed that both the ovaries were of normal size with no observed abnormalities. A contrast-enhanced computed tomography (CT) scan showed large amounts of ascites and omental caking (Fig. 1A). The patient underwent upper gastrointestinal fiberscopy (GF) to investigate the possibility of primary gastric cancer, the most frequent cause of peritoneal dissemination in Japan. A 4-cm submucosal mass without ulceration was detected on the posterior wall of the lesser curvature of the gastric antrum. The lesion was elastic, hard, and covered with an intact mucosa, implying a gastric SMT similar to a gastrointestinal stromal tumor (GIST) (Fig. 1B). The subsequent examinations, including a total colonoscopy, revealed no other primary tumors. Cellblock specimens of ascites showed adenocarcinoma cells that were immunohistochemically positive for BerEP4, PAX-8, WT-1, and p53 (Fig. 2). These findings were consistent with high-grade serous Müllerian adenocarcinoma. Thus, a clinical diagnosis of PPSC, FIGO stage IIIc, was rendered. PPSC appeared life-threatening at the time of diagnosis, so we prioritized systemic chemotherapy for PPSC rather than a gastric biopsy of the SMT. The patient received three cycles of a dose-dense paclitaxel and carboplatin regimen (carboplatin AUC $6 \mathrm{mg}$ / $\mathrm{mL} / \mathrm{min}$ on day 1 and paclitaxel $80 \mathrm{mg} / \mathrm{m}^{2}$ on days 1,8 , and 15). Two months later, a CT scan revealed a reduction in both ascites and the omental tumor, and interval debulking surgery for the tumor was performed. Intra-operative findings demonstrated bloody ascites, a thickened greater omentum, and disseminated tumors less than $10 \mathrm{~mm}$ in the abdominal cavity. The uterus and bilateral adnexae were not affected by the tumor (Fig. 3). The gastric submucosal mass became smaller (3 $\mathrm{cm}$ in diameter) after chemotherapy. Following this, the patient underwent a total abdominal hysterectomy with bilateral salpingo-oophorectomy, partial omentectomy, partial gastrectomy, and resection for the dissemination. The final 


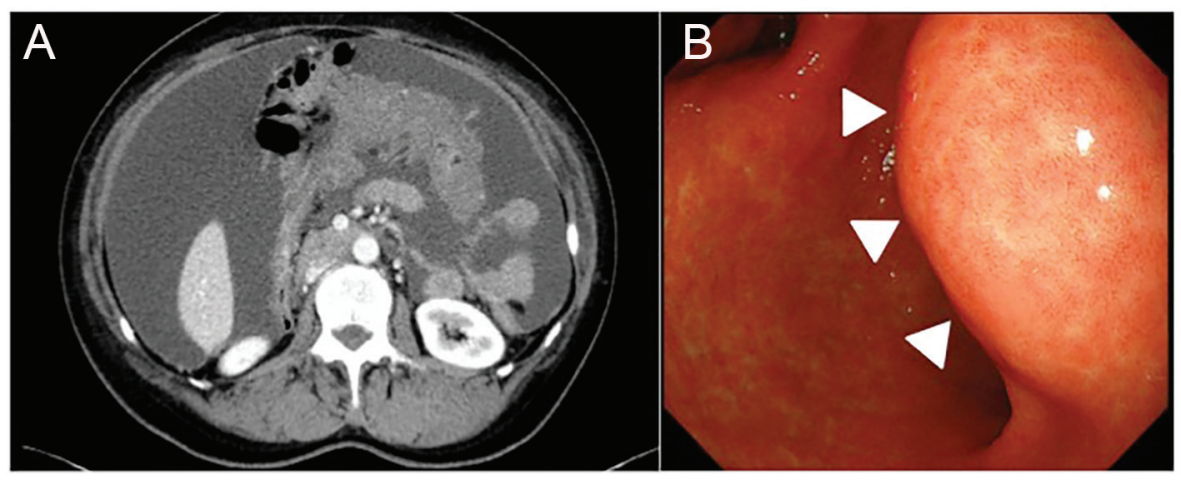

Figure 1. (A) A contrast-enhanced CT scan, showing large amounts of ascites and omental caking. (B) Upper gastrointestinal fiberscope showed a 4-cm submucosal mass without ulceration on the posterior wall of the lesser curvature of the gastric antrum (arrowhead).

pathology of the omental lesion and dissemination was highgrade serous adenocarcinoma (Fig. 4A). Although there was a small amount of adenocarcinoma cells on the right fallopian tube, definite serous tubal intraepithelial carcinoma was not identified. Furthermore, the residual adenocarcinoma was mainly located on the omentum. The gastric lesion showed adenocarcinoma closely resembling peritoneal high-grade serous adenocarcinoma. It was covered with intact gastric mucosa and located in the proper muscular layer of the subserosa (Fig. 4B). These findings strongly support the final diagnosis of PPSC metastasizing to the stomach. The patient was discharged without complications and completed an additional three cycles of chemotherapy. She has been doing well for 6 months without recurrence.

\section{Discussion}

We report a case of gastric metastasis from PPSC mimicking an SMT of the stomach. Gastric metastasis of PPSC is unusual. Based on the similar clinical and histopathological characteristics, PPSC has recently been classified as a pelvic high-grade serous adenocarcinoma. This group also comprises ovarian and tubal serous adenocarcinoma. There are no reports of gastric metastasis from PPSC in the English literature. Previously reported cases of gastric metastasis from pelvic high-grade serous adenocarcinoma are summarized in Table 1 [1-14]. Of these 15 cases, only one Japanese case was reported as PPSC, excluding the case discussed here [14]. However, we cannot dismiss the possibility of more PPSC cases because the ovarian size at the initial diagnosis was not described in the most cases [1-9, 11-13] and another report revealed the size of the ovary was $3 \mathrm{~cm}$ on PET/CT [10]. Of the 15 cases, three cases were symptomatic and detected at the initial diagnosis; the other cases were diagnosed as postoperative recurrence at the stomach. The present case demonstrated that gastric metastasis of PPSC could be asymptomatic, similar to most primary gastric SMTs at the time of first diagnosis.

The mechanism behind gastric metastasis of PPSC remains unclear, although microscopic dissemination seems to be a possible explanation for the present case. This case did not show lymph node metastasis, hematogenous metastasis, or direct invasion to the stomach. However, one of these microscopic disseminations might grow and develop due to an abundant supply of blood from the stomach.

In the diagnostic process of PPSC, gynecologists occasionally need GF to rule out other possible primary malignancies including gastric cancer. When gastroenterologists detect an SMT, primary gastric mesenchymal tumors including GIST, leiomyoma, and schwannoma are listed at the top of the differential diagnosis. However, we should consider the possibility

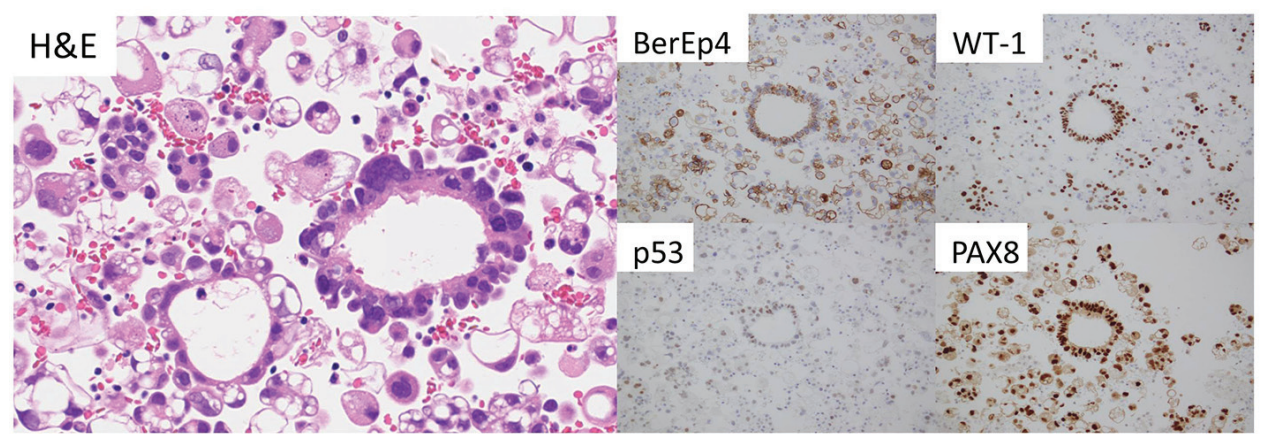

Figure 2. Adenocarcinoma cells in the cellblock specimen (H\&E). These tumor cells show immunoreactivity for BerEP4, WT-1, p53, and PAX-8. 


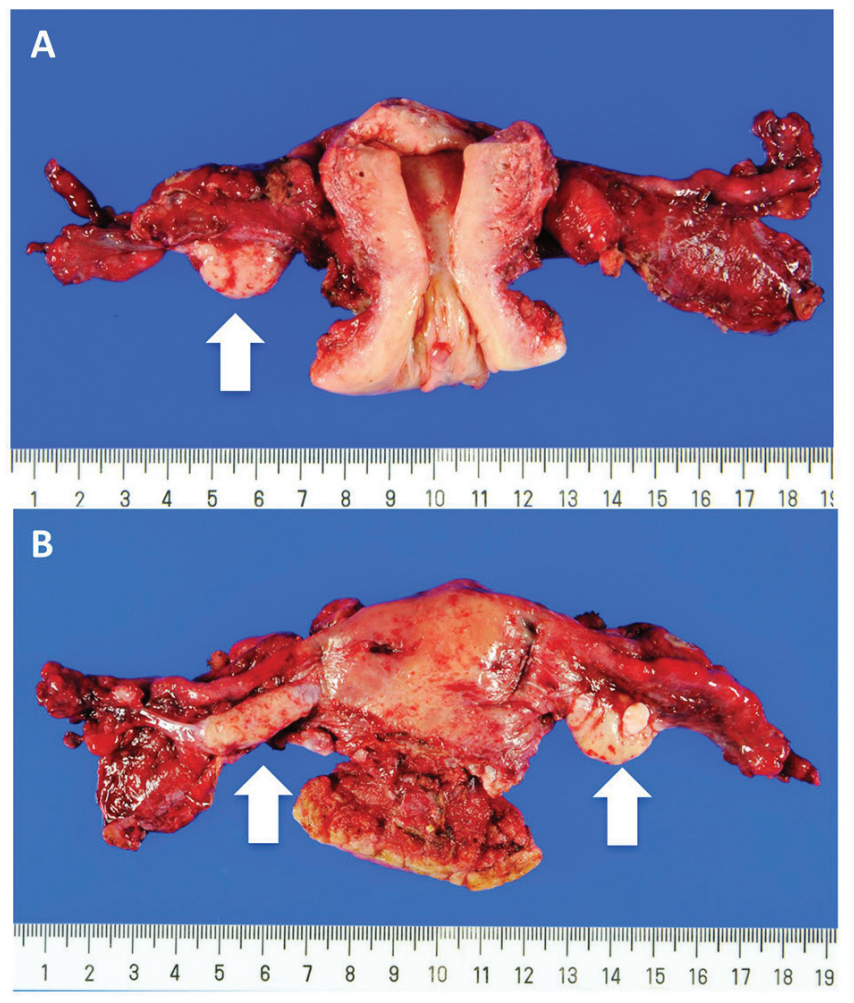

Figure 3. Uterus and bilateral adnexae. (A) Anterior view and (B) Posterior view. Both the ovaries (arrow) and the fallopian tubes are macroscopically intact. of gastric metastasis, which enables us to conduct an adequate debulking surgery. At the same time, unnecessary gastrectomy for benign SMT should be avoided. Thus, if the situation allows, preoperative gastric biopsy is recommended to judge the indication for surgical treatment. Fortunately, a tissue sampling method for gastric SMT has improved recently. Jhala et al reported that the diagnostic accuracy for specimens obtained is $96 \%$ with an endoscopic ultrasound guided fine-needle aspiration biopsy (EUS-FNAB) [15]. For the present case, we prioritized chemotherapy for a life-threatening malignancy and the subsequent apparent reduction in size of the gastric SMT indicated the possibility of metastatic adenocarcinoma. Thus, partial gastrectomy was performed for confirming pathological diagnosis, which resulted in optimal surgery. In addition, Takigawa et al reported they could perform preoperative EUSFNAB and avoid gastrectomy for scar with no residual tumor [14].

In conclusion, we reported the case of gastric metastasis from PPSC. We recommend that when a gastric SMT is detected on GF during the diagnostic process of PPSC, gynecologists should be aware of the possibility of gastric metastasis from PPSC as well as a primary gastric mesenchymal tumor. If the situation allows, preoperative gastric biopsy should be considered to determine an appropriate therapeutic strategy.

\section{Acknowledgments}

We are grateful to Dr. Wada for performing the operation

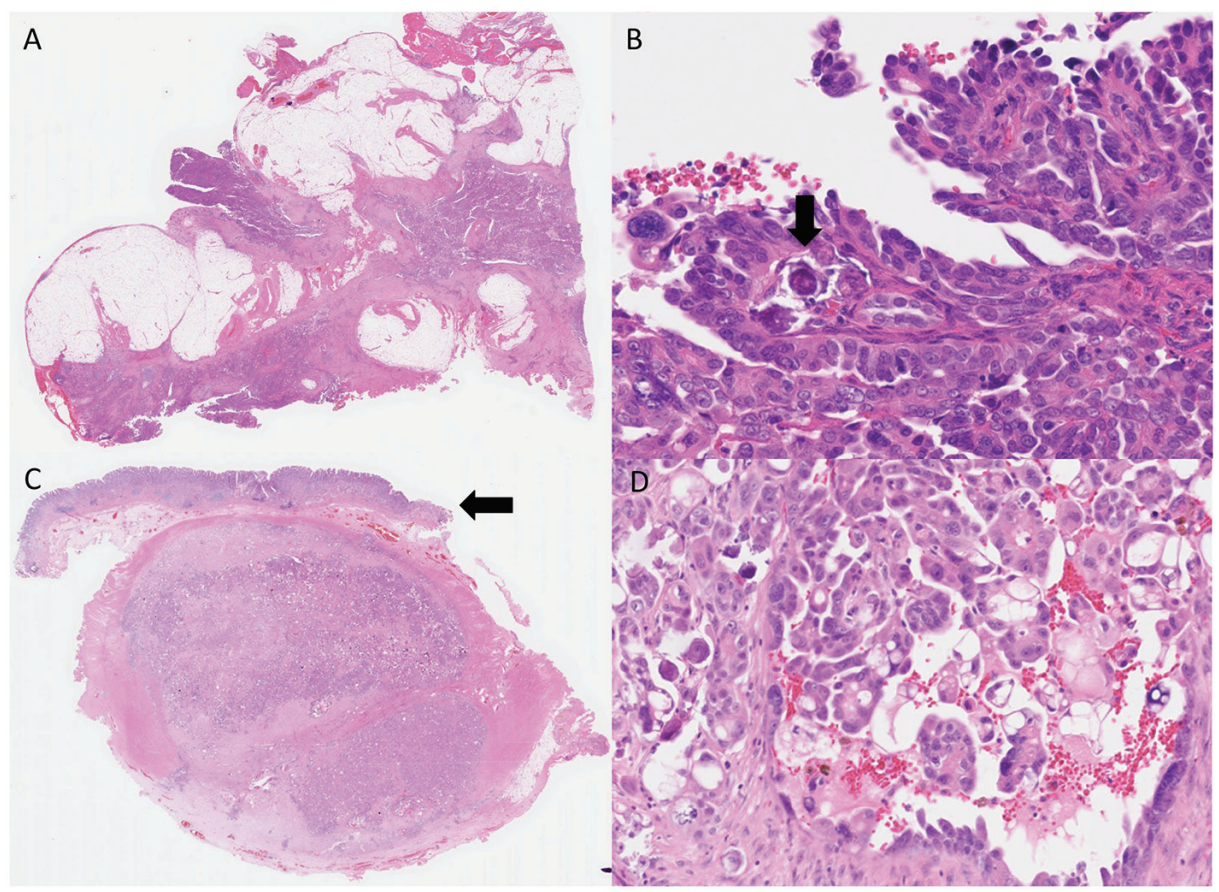

Figure 4. High-grade serous adenocarcinoma (H\&E). (A) High-grade serous adenocarcinoma in the omentum. (B) High-power view, adenocarcinoma cells show nuclear atypia. Psammoma bodies are also observed (arrow). (C) Gastric metastasis from a peritoneal serous adenocarcinoma, covered with intact gastric mucosa (arrow). (D) Adenocarcinoma cells showing similar morphology to those of the omentum. 
Table 1. Summary of Reported Cases of PPSC Metastasizing to the Stomach

\begin{tabular}{|c|c|c|c|c|c|c|}
\hline No. & Age (years) & Primary site & Time of diagnosis & Ovarian size & Survival at Reported FU & Reference \\
\hline 1 & 55 & Ovary & 7 years after $\mathrm{PDS}^{\dagger}$ & $\mathrm{NR}^{\ddagger}$ & $\mathrm{NED}^{\S}$ & {$[1]$} \\
\hline 2 & 42 & Ovary & 18 years after PDS & NR & 12 months NED & {$[2]$} \\
\hline 3 & 62 & Ovary & 10 months after PDS & NR & 6 months DOD & [3] \\
\hline 4 & 48 & Ovary & 21 years after PDS & NR & NR & {$[4]$} \\
\hline 5 & 65 & Ovary & 16 years after PDS & NR & DOD & {$[5]$} \\
\hline 6 & 51 & Ovary & NR & $114 \times 94 \mathrm{~mm}$ & NR & [6] \\
\hline 7 & 49 & Ovary & 4.3 years after PDS & NR & 18 months NED & [7] \\
\hline 8 & 70 & Ovary & First diagnosis & NR & NR & [8] \\
\hline 9 & 61 & Ovary & 7 months after PDS & NR & 18 months DOD & [9] \\
\hline 10 & 55 & Ovary & First diagnosis & $3 \mathrm{~cm}$ & 12 months NED & {$[10]$} \\
\hline 11 & 61 & Ovary & 12 years after PDS & NR & 5 months NED & {$[11]$} \\
\hline 12 & 58 & Ovary & 3 years after PDS & NR & NR & {$[12]$} \\
\hline 13 & 73 & Ovary & 7 years after PDS & NR & 10 months DOD & [13] \\
\hline 14 & 64 & Peritoneal carcinoma & First diagnosis & Normal size & 18 months NED & {$[14]$} \\
\hline 15 & 62 & Peritoneal carcinoma & First diagnosis & Normal size & 6 months NED & Present case \\
\hline
\end{tabular}

†Primary debulking surgery. ${ }^{\ddagger}$ Not reported. §No evidence of disease. โDead of disease.

with us.

\section{Conflicts of Interest}

All authors declare no conflicts of interest.

\section{References}

1. Sangha S, Gergeos F, Freter R, Paiva LL, Jacobson BC. Diagnosis of ovarian cancer metastatic to the stomach by EUS-guided FNA. Gastrointest Endosc. 2003;58(6):933935.

2. Pernice M, Manci N, Marchetti C, Morano G, Boni T, Bellati F, Panici PB. Solitary gastric recurrence from ovarian carcinoma: a case report and literature review. Surg Oncol. 2006;15(4):267-270.

3. Taylor RR, Phillips WS, O'Connor DM, Harrison CR. Unusual intramural gastric metastasis of recurrent epithelial ovarian carcinoma. Gynecol Oncol. 1994;55(1):152155.

4. Wallace W, Mulholland K, Epanomeritakis E. Bleeding gastric varices - a rare complication of ovarian cancer. Int J Clin Pract. 2005;59(1):119-120.

5. Dupuychaffray JP, Auger C, Funes De La Vega M, Riche A, Boulanger V, Blanchot P. [Gastric metastasis from ovarian carcinoma revealed by a gastro-splenic perforation]. Gastroenterol Clin Biol. 2004;28(5):490-493.

6. Bechade D, Desrame J, Raynaud JJ, Eggenspieler P, Baranger $\mathrm{B}$, Vedrine $\mathrm{L}$, Ceccaldi $\mathrm{B}$, et al. [Hemorrhagic gastric metastasis of an ovarian carcinoma]. Gastroenterol Clin Biol. 2005;29(10):1065-1066.

7. Jung HJ, Lee HY, Kim BW, Jung SM, Kim HG, Ji JS,
Choi H, et al. Gastric Metastasis from Ovarian Adenocarcinoma Presenting as a Submucosal Tumor without Ulceration. Gut Liver. 2009;3(3):211-214.

8. Carrara S, Doglioni C, Arcidiacono PG, Testoni PA. Gastric metastasis from ovarian carcinoma diagnosed by EUS-FNA biopsy and elastography. Gastrointest Endosc. 2011;74(1):223-225.

9. Majerus B, Timmermans M. [Gastric metastases of ovarian adenocarcinoma. Apropos of a case]. Acta Chir Belg. 1990;90(4):166-171.

10. Kang WD, Kim CH, Cho MK, Kim JW, Lee JS, Ryu SY, Kim YH, et al. Primary epithelial ovarian carcinoma with gastric metastasis mimic gastrointestinal stromal tumor. Cancer Res Treat. 2008;40(2):93-96.

11. Zhou JJ, Miao XY. Gastric metastasis from ovarian carcinoma: a case report and literature review. World J Gastroenterol. 2012;18(43):6341-6344.

12. Kim EY, Park CH, Jung ES, Song KY. Gastric metastasis from ovarian cancer presenting as a submucosal tumor: a case report. J Gastric Cancer. 2014;14(2):138-141.

13. Hwangbo S, Kwon OK, Chung HY, Yu W. Improved Survival of a Patient with Gastric and Other Multiple Metastases from Ovarian Cancer by Multimodal Treatment: A Case Report. J Gastric Cancer. 2015;15(3):218-221.

14. Takigawa H, Furudoi A, Kan H, Nonaka M, Fujimoto Y, Komatsu H, Tokumo H, et al. A case of primary peritoneal carcinoma with gastric metastasis that was diagnosed by endosonography-guided fine needle aspiration. Gastroenterological Endoscopy. 2013;55(11):3562-3567.

15. Jhala NC, Jhala D, Eltoum I, Vickers SM, Wilcox CM, Chhieng DC, Eloubeidi MA. Endoscopic ultrasound-guided fine-needle aspiration biopsy: a powerful tool to obtain samples from small lesions. Cancer. 2004;102(4):239246. 